\title{
Huntington Disease like 2 (HDL-2) with parkinsonism and abnormal DAT-SPECT - a novel observation
}

Eoin Mulroy ${ }^{1}$, Anna Latorre ${ }^{1}$, Elisa Menozzi1 ${ }^{1}$, Pei Chiek Teh ${ }^{1}$, Francesca Magrinelli ${ }^{1,2}$, Kailash P Bhatia ${ }^{1}$

${ }^{1}$ Department of Clinical and Movement Neurosciences, UCL Queen Square Institute of Neurology, London WC1N 3BG

${ }^{2}$ Department of Neurosciences, Biomedicine, and Movement Sciences, University of Verona, Verona, Italy

\section{Corresponding author:}

Eoin Mulroy

Department of Clinical and Movement Neurosciences, UCL Queen Square Institute of Neurology, London WC1N 3BG

eoin.mulroy@nhs.net

Phone: +4442034567890

Word count: 712

Key words: Chorea; Parkinsonian disorders; trinucleotide repeat expansion 


\section{Introduction}

Huntington's disease-like 2 (HDL-2) is an autosomal dominant neurodegenerative disorder caused by a CTG/CAG trinucleotide repeat expansion ( $\geq 40$ repeats) in the junctophilin-3 gene on chromosome 16q24.3 [1]. The affected protein product, junctophilin-3, plays a role in anchoring the endoplasmic reticulum to the plasma membrane. The disorder generally manifests in mid-life as a Huntington's disease (HD) phenocopy syndrome comprising cognitive decline, neuropsychiatric manifestations and movement disorders (generally chorea)[2, 3]. Most exhibit oculomotor abnormalities, and MRI brain frequently shows caudate atrophy [3]. To-date, the disorder is reported exclusively in individuals with African ancestry, in which it is the most common HD phenocopy syndrome[3]. We describe a patient with geneticallyconfirmed HDL-2 exhibiting parkinsonism alongside chorea and cognitive decline, and in whom dopamine transporter single photon emission computed tomography (DAT-SPECT) imaging was abnormal.

\section{Case report}

A 51 year-old woman of Afro-Caribbean descent was reviewed in the movement disorder clinic. She was unaware of any problems, but her brother reported gradual worsening in balance, memory and speed of movement for 6 years. Over a similar time period, a number of involuntary 'twitchy' movements had also appeared. Her mother was said to have exhibited similar symptoms in her 30s and died aged 53 years. She had two brothers, one of whom was well, and one who had a similar clinical picture beginning in his late 30 s. 
Examination showed slight right torticollis and dystonic finger posturing. She had difficulty with voluntary saccade initiation and difficulty maintaining fixation. There was mild generalized chorea. Bilateral asymmetric rigidity and bradykinesia was evident. She walked with a broad base and reduced arm swing (video 1). DAT-SPECT imaging confirmed bilateral asymmetric reduction in striatal tracer uptake on both qualitative and quantitative analysis (figure 1). The patient refused MRI imaging, and did not desire treatment for either parkinsonism or chorea.

Given the clinical picture, dominant inheritance pattern and Afro-Caribbean ancestry, southern blot analysis for an expansion at the JPH3 locus was performed, confirming one allele with a CAG trinucleotide expansion in the pathogenic range (52 repeats).

\section{Discussion}

There are two principal learning points from this case. The first relates to the phenotypic diversity of the HDL-2 clinical syndrome. While HD-like presentations with psychiatric and cognitive dysfunction accompanied by choreiform movement are most common, parkinsonism, dystonia and myoclonus may all form part of the movement disorder phenomenology [4, 5]. 
Parkinsonism can in fact be the predominant movement disorder, especially in patients with longer repeat lengths [5-7].

Second, this is, to our knowledge, the first report of dopamine transporter imaging findings in a case of HDL-2. The observed reduction in striatal DAT uptake on SPECT imaging is akin to that occasionally seen in HD [8], and confirms a pre-synaptic component to striatal dopaminergic deficiency in HDL-2. Whether this finding has therapeutic implications e.g. levodopa responsiveness, in those patients with HDL-2 related parkinsonism, remains to be determined.

Given the paucity of neuropathological, neurochemical and functional imaging studies in the HDL-2 population, one can only speculate on the aetiopathologic significance of reduced striatal DAT ligand uptake in our case. Pre-synaptic dopaminergic degeneration secondary to nigral cell loss, reduced striatal dopaminergic nerve terminal density and dysfunctional synthesis, transport and/or expression of the dopamine transporter on striatal dopaminergic terminals are all potential explanations. In this regard, neuropathologic studies in HDL-2 (and indeed in HD) demonstrate preserved nigral cell populations, suggesting that nigral cell loss akin to that seen in, for example, Parkinson's disease, is less likely to account for the observed abnormalities on DAT imaging [9]. Functional neuroimaging studies in HD confirm that the majority of nigrostriatal deficits are post-synaptic in nature, with pre-synaptic involvement likely occurring more so in advanced disease [10]. A Drosphila model of HDL-2 failed to demonstrate significant impairments in axonal transport (in contrast to those seen in HD), which may render less likely a defect in DAT transport to 
striatal terminals [11]. There is evidence in the HD literature of reduced presynaptic striatal dopaminergic terminal density, in proportion to the degree of striatal atrophy [12]-if similar changes were present in HDL-2, this could account for the observed changes on DAT imaging.

\section{Conclusion}

HDL-2 should be borne in mind as a cause of parkinsonism, especially if it presents alongside cognitive/psychiatric symptoms, hyperkinetic movements, or in those with African ancestry. DAT-SPECT imaging can be abnormal in HDL-2.

\section{Ethics statement:}

All procedures followed were in line with the journal's ethics policy and written consent was obtained from all patients

\section{Funding:}

This research did not receive any specific grant from funding agencies in the public, commercial, or not-for-profit sectors. 


\section{References}

1. Holmes SE, O’Hearn E, Rosenblatt A, Callahan C, Hwang HS, IngersollAshworth RG, Fleisher A, Stevanin G, Brice A, Potter NT, Ross CA, Margolis RL. A repeat expansion in the gene encoding junctophilin-3 is associated with Huntington Disease-like 2. Nat Genet. 2001;29:377-378.

2. Anderson DG, Walker RH, Connor M, Carr J, Margolis RL, Krause A. A Systematic Review of the Huntington Disease-Like 2 Phenotype. J Huntingtons Dis. 2017;6(1):37-46. doi: 10.3233/JHD-160232.

3. Anderson DG, Krause A, Margolis RL. Huntington Disease-Like 2. 2004 Jan 30 [Updated 2019 Jun 27]. In: Adam MP, Ardinger HH, Pagon RA, et al., editors. GeneReviews ${ }^{\circledR}$ [Internet]. Seattle (WA): University of Washington, Seattle; 1993-2019. Available from: https://www.ncbi.nlm.nih.gov/books/NBK1529/

4. Walker RH, Jankovic J, O'Hearn E, Margolis RL. Phenotypic features of Huntington's disease-like 2. Mov Disord 2003;18: 1527-1530.

5. Bardien S, Abrahams F, Soodyall H, van der Merwe L, Greenberg J, Brink T, Carr J. A South African mixed ancestry family with Huntington diseaselike 2: Clinical and genetic features. Mov Disord. 2007;22:2083-2089.

6. Margolis RL. Huntington Disease-Like 2. GeneReviews. 2012 http://www.ncbi.nlm.nih.gov/books/NBK1529/

7. Krause A, Mitchell C, Essop F, et al. Junctophilin 3 (JPH3) expansion mutations causing Huntington disease like 2 (HDL2) are common in South African patients with African ancestry and a Huntington disease 
phenotype. Am J Med Genet B Neuropsychiatr Genet. 2015;168(7):573585. doi:10.1002/ajmg.b.32332

8. Gamez J, Lorenzo-Bosquet C, Cuberas-Borrós G, Carmona F, HernándezVara J, Castilló J, Castell-Conesa J. Does reduced [(123)I]-FP-CIT binding in Huntington's disease suggest pre-synaptic dopaminergic involvement? Clin Neurol Neurosurg. 2010 Dec;112(10):870-5. doi:10.1016/j.clineuro.2010.07.014. Epub 2010 Aug 19.

9. Rudnicki DD, Pletnikova O, Vonsattel JP, Ross CA, Margolis RL. A comparison of huntington disease and huntington disease-like 2 neuropathology. J Neuropathol Exp Neurol. 2008 Apr;67(4):366-74. doi: 10.1097/NEN.0b013e31816b4aee.

10. Hwang WJ, Yao WJ. SPECT study of the nigrostriatal dopaminergic system in Huntington's disease. J Neuroimaging. 2013 Apr;23(2):192-6. doi: 10.1111/j.1552-6569.2011.00671.x.

11. Krench M, Cho RW, Littleton JT. A Drosophila model of Huntington disease-like 2 exhibits nuclear toxicity and distinct pathogenic mechanisms from Huntington disease. Hum Mol Genet. 2016 Aug 1;25(15):3164-3177. doi: 10.1093/hmg/ddw166. Epub 2016 Jun 10.

12. Ferrante RJ, Kowall NW. Tyrosine hydroxylase-like immunoreactivity is distributed in the matrix compartment of normal human and Huntington's disease striatum. Brain Res. 1987 Jul 21;416(1):141-6. 


\section{Figure legend}

Figure 1: DAT-SPECT imaging showing bilateral reduced tracer activity in both striata, manifesting as reduced striatal to background contrast. The changes are more pronounced in the right putamen.

\section{Captions:}

Supplementary material 1: Video of the patient showing generalized chorea, bilateral asymmetric parkinsonism and difficulty with saccade initiation (frequently initiated with head thrust) 